Doug Geisler, Eva K. Grebel, and Dante Minniti, eds.

\title{
Near-Infrared Imaging of the LMC Nebula N11B
}

\author{
Rodolfo H. Barbá \\ Observatorio Astronómico La Plata, B1900FWA, La Plata, Argentina \\ Mónica Rubio \\ Departamento de Astronomía, Universidad de Chile, Casilla 36-D, \\ Santiago, Chile
}

\begin{abstract}
We present new near-infrared observations of the N11B nebula in the LMC. The presence of IR sources with intrinsic IR excess, a methanol maser and a very prominent PDR regions suggest strong star forming activity in N11B.
\end{abstract}

\section{Introduction}

The N11 nebular complex is the second largest H II region in the Large Magellanic Cloud after 30 Doradus Nebula, and it consists of a huge bubble surrounded by nine distinct nebular entities. In the center of the complex the OB association LH9 is located. It is dominated by the massive compact cluster HD 32228 with a WC4 star of about 3.5 Myr (Walborn et al. 1999). Around LH9 there are nother younger $\mathrm{OB}$ associations embedded in dense nebular regions, two of which have O3 stars among their members: LH13 in N11C and LH10 in N11B. Walborn \& Parker (1992) proposed a two-stage starburst scenario to explain the morphological distribution of $\mathrm{OB}$ associations in N11, where the initial and central burst (in LH9) maybe triggered a second generation burst about $2 \times 10^{6}$ years later around the neighbor molecular clouds. This burst is now characterized by the presence of O3 stars. Also, N11 is a large molecular complex composed of at least 21 separate clouds (Israel \& de Graauw 1991). The CO emission peaks correlate with $\mathrm{H} \alpha$ and FIR dust emissions suggesting star formation activity in the nebula (Caldwell \& Kutner 1996).

Thus, the N11 region is a very good candidate to study sequential star formation processes in a giant $\mathrm{H}$ II region, and how the different elements (gas, stars and dust) are related. We present new near-infrared observations of N11B and compare them with HST optical images to provide further evidence about the ongoing star forming activity in regions of this kind.

\section{Discussion}

Near-infrared images in $J, H$, and $K s$ bands of the LH10 association were obtained at Las Campanas Observatory (Chile) in January 1993, using the nearIR camera IRCAM attached to the $2.5-\mathrm{m}$ DuPont Telescope. The images covered 
an area of $110^{\prime \prime}$ square, centered in the O6 V star PGMW 3070 (Parker et al. 1992), and show a cluster of blue stars with relatively low extinction. At $60^{\prime \prime}$ to the North of PGWM 3070, and around PGMW 3123 (O8.5 V) a nebular feature appears with several embedded IR sources. Figure 1 shows a comparison of this nebulosity in Ks and the F656N $(\mathrm{H} \alpha)$ WFPC2 images. This nebular feature is a photo-dissociation region (PDR) between the molecular cloud and the ionized cavity. Several IR sources are embedded in the dusty prominence, and also it contains a maximum in the CO distribution. The PDR interface is easily seen in the HST images and that shows a rugged surface with many dark and bright protuberances, almost all of them pointing to the hot stars. The PDR emission pattern with striations pointing normal to the interface looks like the heads of pillars in M16 (Hester et al. 1996). Figure 1 shows that several IR sources are identified with small finger-like dusty structures, suggesting the presence of embedded protostars. A methanol maser emission (Ellingsen et al. 1994) is associated with the N11B nebula, and it is located inside the dusty prominence. Three stellar sources are inside the 2.5 ATCA beam. One of these sources is the O8.5 V star PGMW 3123, and the other two are IR sources (IRS 147 and 148, Barbá et al. 2001). Both IR stellar-like sources are among the reddest sources in the field. Some of these IR sources with intrinsic IR excess are candidates to intermediate-mass Herbig Ae/Be stars.

\section{Conclusions}

The morphological picture of the N11B nebula suggests that a second generation of stars in the N11 nebular complex is still forming now (as proposed by Walborn \& Parker 1992). The N11B region surveyed with IR images have several YSO candidates. The dusty prominence around PGMW 3123 is the prime site for star formation in N11B, with several IR embedded sources and a methanol maser. For the first time a methanol maser was directly associated with IR stellar sources outside the Milky Way.

Acknowledgments. RHB thanks the Fundación Antorchas (Argentina) for supporting this work. MR wishes to acknowledges support from Fondecyt (Chile) grant No. 1990881 and No. 7990042.

\section{References}

Barbá, R.H., Rubio, M., Roth, M.M., García, J. 2001, in preparation

Caldwell, D.A., \& Kutner, M.L. 1996, ApJ, 472, 611

Ellingsen, S.P., et al. 1994, MNRAS, 269, 1019

Hester, J.J., et al., 1996, AJ, 111, 2349

Israel, F.P., \& de Graauw, T., IAU Symp., 148, 45

Parker, J.Wm., Garmani, C.D., Massey, P., \& Walborn, N.R., 1992, AJ, 103, 1205

Walborn, N.R., et al. 1999, AJ, 118, 1684

Walborn, N.R., \& Parker, J.Wm., 1992, ApJ, 399, L87 

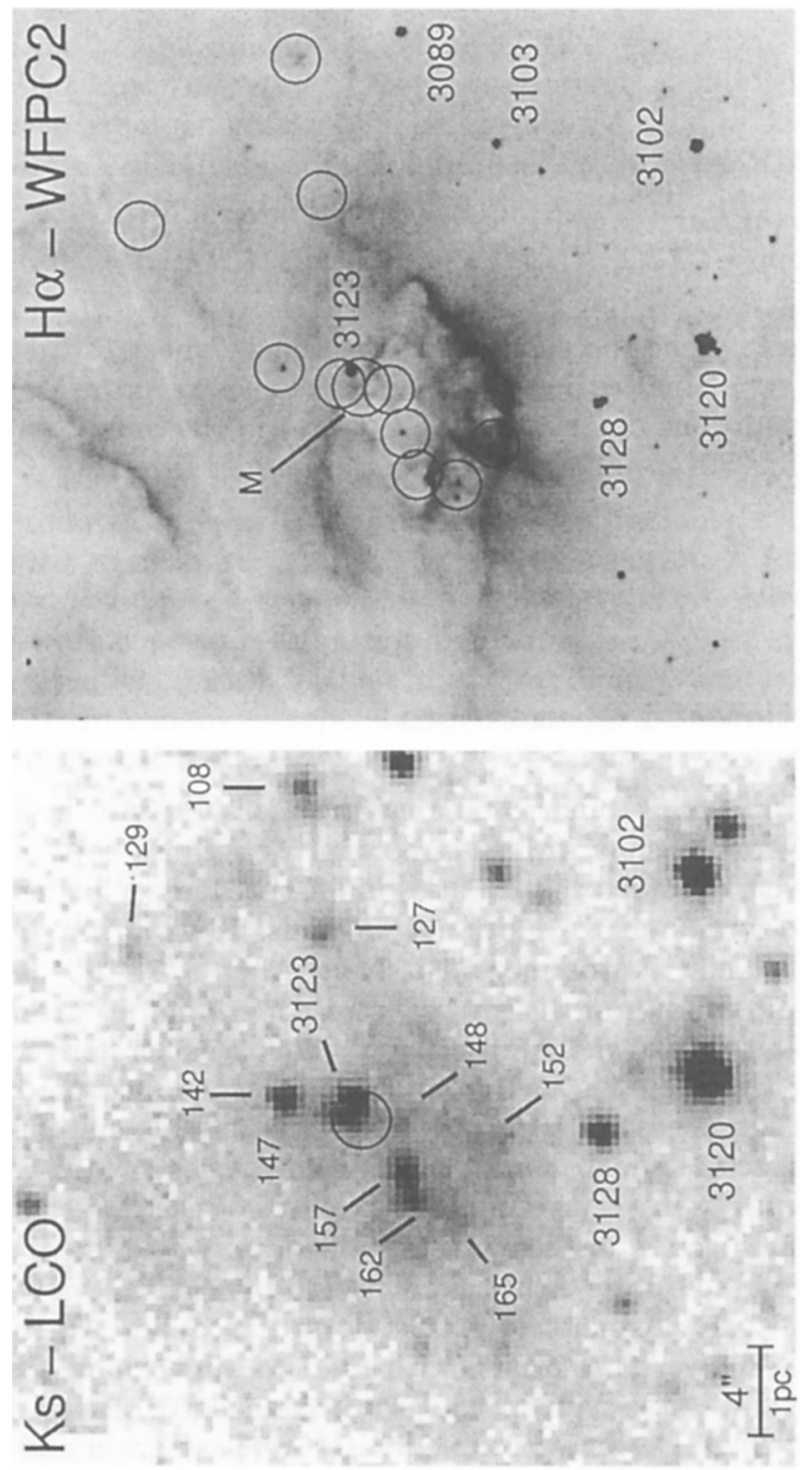

Figure 1. Lower panel: Section of the $K s$ band image around the dusty prominence in N11B. Big numbers are from optical photometry of Parker et al. (1992), small numbers are from NIR photometry of Barbá et al. (2001). The methanol maser position is marked with a circle. Upper panel: Section of the F656N ( $\mathrm{H} \alpha)$ WFPC2 image of the same field. Some IR sources are marked with circles. The methanol maser is also indicated by an " $\mathrm{M}$ ". 